still be triaged and seen. A clear referral with photographs will greatly aid this process. Biopsy under local anaesthesia and axial imaging remain available and virtual multi-disciplinary team meetings are running. Surgery involving free tissue transfer is extremely limited at present. However, if a tumour is detected at an early stage then wide local excision even under local anaesthesia is possible.

Sadly, mouth cancer is a condition that will be affected by the coronavirus pandemic. Only time will tell the true nature and enormity of this impact.

$$
\begin{array}{r}
\text { M. A. O. Lewis, C. V. Thomas, Cardiff, UK } \\
\text { https://doi.org/10.1038/s41415-020-1591-x }
\end{array}
$$

\section{Don't forget about head and neck cancer}

Sir, during the current virus situation there is a real risk that patients with malignant lesions present late to specialist teams, potentially resulting in worsened outcomes. Telephone consultation is available to patients via their GDP and offers an opportunity to identify high risk lesions and organise their timely onward referral if appropriate. Strict triage is essential and must include screening questions relating to head and neck oral squamous cell carcinoma.

In 2015, Birur et al. found remote monitoring of potentially cancerous oral lesions by primary care practitioners to have improved early detection and ultimate diagnosis of oral cancer. ${ }^{1}$ A similar system could be adapted in the current health climate, with patients taking photos of lesions found in their own mouth (assisted perhaps by a household member) and emailing them to their dental practice for review. In order to ensure the oral cavity is imaged satisfactorily, perhaps video may be an option, online facilities whereby a practitioner could ask the patient to move their tongue, swallow, or improve the angle of view to enable more detailed assessment.

When a fast track referral is received by OMFS, it can be reviewed by an experienced member of the team. If the referring clinician is able to obtain any photographs from the patient, they may be used, in addition to an ENT UK validated telephone risk stratification system which enables an evidence-based risk assessment to be made. ${ }^{2}$ Ongoing management of potential cases, whilst challenging, can then be arranged according to this risk.
We implore dental professionals to continue their rigorous efforts to detect oral cancer, by asking patients to phone in to their practice if they detect unexplained oral ulceration which persists longer than three weeks, a persistent lump in their neck, or notice a red or white patch anywhere in the mouth. Additional red flag symptoms would include changes to the voice, unintended weight loss and pain or difficulty swallowing. Despite the current uncertainty, our duty to patients still involves aiding their referral to specialist services should it become necessary.

\section{R. Taylor, E. Walshaw, A. Kanatas, Leeds, UK}

\section{References}

1. Birur P N, Sunny S P, Jena $S$ et al. Mobile health application for remote oral cancer surveillance. J Am Dent Assoc 2015; 146: 886-894.

2. ENT UK 2WW Telephone Triage: Service Evaluation INTEGRATE. Available at: https://entintegrate.co.uk/ entuk2wwtt (accessed 17 April 2020).

https://doi.org/10.1038/s41415-020-1592-9

\section{A potential third infection route}

Sir, so far, we are aware of two main routes of transmission SARS-Cov-2: infection by contact with a contaminated object and inhalation of droplets emitted by sneezes and coughs. However, there may be a third infection route of microdroplets, which can remain in the air for perhaps 20 minutes or more, particularly where ventilation is poor. Recent experiments have been undertaken in the Kyoto Institute of Technology and the Japanese Association for Infectious Disease. Laser beams and high sensitivity cameras, which can capture microdroplets 0.01 micrometres in width, have been used to analyse the aftermath of sneezes and coughs. Droplets fall relatively quickly, but small particles (less than 10 micrometres in width) can remain in the air for prolonged periods.

Similar experiments have been undertaken assessing close range conversations where microdroplets can be seen generated during speech, particularly loud conversational speech or heavy breathing (such as after a jog). Stagnation of microdroplets can be minimised with good ventilation and increased air circulation. It is not known what volume of microdroplets can lead to infection, but the possibility that such microdroplets may transmit the virus cannot be ruled out. The risk is that it may be spread by speaking to someone, or potentially being in an area where others have been speaking, particularly if ventilation is poor, and masks are not being worn. Consider the areas in hospitals where most conversations take place, such as offices or corridors, many with inadequate or no ventilation. The implications of this data will become clearer as our understanding improves, but in the meantime, extra caution may be better than the alternative.

F. B. Naini, London, UK https://doi.org/10.1038/s41415-020-1593-8

\section{Saliva testing for COVID-19?}

Sir, Reverse Transcription-Polymerase Chain Reaction (RT-PCR) is the most commonly used molecular diagnostic test for the detection of COVID-19 in biological samples but no universally accepted test is currently available, with several countries adopting different test strategies. ${ }^{1}$ The selection of proper location/test site for sample collection is very important to obtain reliable test results, the most commonly used being naso- and/or oropharynx swabs (NOS). ${ }^{2}$

Although these are relatively easy to collect and test results are highly sensitive, there are limitations related to sample collection and healthcare personnel safety. However, use of saliva as an alternative to NOS for detection of COVID-19 has been suggested. ${ }^{3,4}$ Using saliva samples has a number of 'clinical advantages'; it is less invasive and more convenient to patients as compared to NOS or blood samples $^{4}$ (especially desirable in multiple testing for disease monitoring). Secondly, with clear instructions, patients can collect saliva themselves, thereby minimising the risk of virus transmission to healthcare personnel and avoiding use of personal protective equipment.

This potential use of saliva seems scientifically reasonable as it has been shown to contain live COVID-19 viruses ${ }^{3}$ possibly containing a pool coming from the lower respiratory tract, nasopharynx and infected salivary glands (for some of the coronaviruses, infection of salivary glands occurs very early in the disease process). ${ }^{4}$ Unlike the other SARS virus diseases, the content of salivary COVID-19 (viral load) has been shown to be highest during the first week after symptom onset. ${ }^{5}$ This emphasises the role of saliva as a potential source of viral transmission and, as it could be detected in the saliva as long as 25 days after the onset of symptoms, suggesting its potential use for monitoring viral clearance. ${ }^{5}$ 
Besides RT-PCR-based tests, serological (enzyme immunoassays) diagnostic tests aimed at detecting $\operatorname{IgM}$ and IgG antibodies against the viral antigens are robust methods to diagnose current and past infections. Preliminary studies using serum samples have shown promising results for COVID-19., ${ }^{5,6}$ The production of SARS-CoV-specific secretory IgA in the saliva of animal models was previously shown. ${ }^{7}$ Hence, it is reasonable to speculate that anti-COVID-19 antibodies might also be present in human saliva although this has not been reported nor has their potential use for diagnostics and disease monitoring. This clearly warrants future studies.

Studies are needed to analyse the sensitivity and specificity of saliva based COVID-19 tests before they could be made available as a convenient and cost-effective diagnostic method. Since the presence of live COVID-19 in saliva identifies it as a potential source of viral transmission any collected saliva samples must be handled with care to avoid spilling and spreading of live viruses.

D. Sapkota, S. B. Thapa, Oslo, Norway, B. Hasséus, Gothenburg, Sweden, J. L. Jensen,

Oslo, Norway

\section{References}

1. Pang J, Wang M X, Ang I Y H et al. Potential rapid diagnostics, vaccine and therapeutics for 2019 novel coronavirus (2019-nCoV): A systematic review. J Clin Med 2020; 9: 623.

2. Xie C, Jiang L, Huang $G$ et al. Comparison of different samples for 2019 novel coronavirus detection by nucleic acid amplification tests. Int J Infect Dis 2020; 93: 264-267.

3. To K K-W, Tsang O T-Y, Yip C C-Y. et al. Consistent detection of 2019 novel coronavirus in saliva. Clin Infect Dis 2020; ciaa149. doi:10.1093/cid/ciaa149.

4. Sabino-Silva R, Jardim A C G, Siqueira W L. Coronavirus COVID-19 impacts to dentistry and potential salivary diagnosis. Clin Oral Investig 2020; 24: 1619-1621.

5. To K K-W, Tsang O T-Y, Leung W-S et al. Temporal profiles of viral load in posterior oropharyngeal saliva samples and serum antibody responses during infection by SARS-CoV-2: an observational cohort study. Lancet Infect Dis 2020; S1473-S3099. doi: 10.1016/ S1473-3099(20)30196-1.

6. Guo L Ren L, Yang S et al. Profiling early humoral response to diagnose novel coronavirus disease (COVID19). Clin Infect Dis; 2020; doi:10.1093/cid/ciaa310.

7. Lu B, Huang Y, Huang L et al. Effect of mucosal and systemic immunization with virus-like particles of severe acute respiratory syndrome coronavirus in mice. Immunology 2010; 130: 254-261.

https://doi.org/10.1038/s41415-020-1594-7

\section{Redeployment positives}

Sir, with the coronavirus situation many DCTs have been redeployed to medical specialities including Accident and Emergency, General Medicine and ITU. The roles of these redeployed dentists are now similar to that of junior doctors, which include cannulation and venepuncture, discussion of treatment plans and general ward duties. Understandably, there was anxiety over the readiness and preparedness to assist on medical wards from DCTs. However, the overwhelming response has been that of support, understanding and gratitude from our medical colleagues.

A positive, even in this time of crisis, is that redeployment has allowed DCTs to gain experiences far beyond their normal day to day scope, gaining a better understanding of emergency medicine, dealing with crisis and managing chronically and critically unwell patients. A better understanding of the 'medical side' of dentistry, gives us extended skills, which will be transferred to our future careers. Additionally, DCTs have contributed their own specialist knowledge, improving patient outcomes and reducing the general strain on the NHS. Examples include managing dental trauma, diagnosing and managing acute dental pain of in-patients and managing soft tissue lacerations on wards. This contribution from DCTs has helped to develop a mutual respect and understanding of the roles of both medics and dentists, highlighting the value of dentistry within healthcare. I would like to express my pride and admiration for those redeployed to the NHS frontline. In this time of unprecedented crisis all DCTs across the UK have stepped up to the mark and excelled in supporting our NHS.

S. McLean, Liverpool, UK https://doi.org/10.1038/s41415-020-1595-6

\section{Check-ins not check-ups}

Sir, in these uncertain times, the NHS has faced immense pressure. In response, many of us have been upskilled and redeployed to unfamiliar clinical settings in this unique fight against COVID-19. However, those awaiting redeployment, those self-isolating vor shielded members who cannot work clinically due to underlying health conditions, have a very valuable skill set. We are strong communicators and share an instinctive compassion for our communities. We also carry a moral duty of care for the public and have received training in safeguarding.

These qualities are ideally suited to befriending. Befriending offers supportive, reliable relationships to individuals who are lonely or socially isolated. Maintaining our mental health and wellbeing is so important during this time of social distancing. As we know well, we all need other people, but not everyone has someone. Befriending during COVID-19 involves contacting service users at an arranged time and chatting, listening and ensuring they are coping well. Instead of providing our usual check-ups, we could be checking in. We could be giving our time to others, arguably the most valuable of all assets.

Charities offering befriending services have fast track online modules to provide immediate training. As healthcare professionals we all have Disclosure and Barring Certificates already, which further accelerates our application. Diane Claridge from Befriending Networks believes that charities would be very grateful for any support our profession can offer. She recommends visiting www.befriending.co.uk, searching the directory for local charities and contacting them directly to get involved. Examples of charities that support befriending include Age UK, Independent Age, Royal Voluntary Service (RVS) and Re-engage (formerly Contact the Elderly).

Oyovwe Kigho is the founder of the Widows Empowerment Trust. Oyovwe has recently reported a huge increase in the number of vulnerable widows seeking the support of her charity. We are befrienders with the Widows Empowerment Trust and have completed the online training. Providing this service is incredibly rewarding. So, in a time when negativity and fear are in the air, let us remember the positivity and support we can bring to others.

F. Loy, L. Reynolds, Manchester, UK https://doi.org/10.1038/s41415-020-1596-5

\section{Call for help}

Sir, I wonder if any of your readers could help at all. I am currently unemployed and on a 'gap year' of sorts whilst applying for speciality training. In fact, I am actually meant to be travelling around South America as I type this, however due to the obvious I am not. I am lucky enough to be living with my parents for the foreseeable so can use my travel savings to pay for the minimal bills and outgoings I have. What I would like to be able to do is work or help in any way possible with the fight against COVID-19. Dental core trainees and general dental practitioners are being redeployed onto wards and to Urgent Dental Care centres, but 